\title{
An unusual case of Rhodotorula mucilaginosa fungaemia in a cancer patient
}

\author{
Valentino Granero, ${ }^{1}$ Elvio Peyronel, ${ }^{1}$ Nicoletta Mensa, ${ }^{2}$ Nicola Liuzzi, ${ }^{2}$ Cristina Costa, ${ }^{3}$ Maria Rita Cavallo \\ ${ }^{1}$ Rivoli-Pinerolo Unified Analysis Laboratory, ${ }^{2}$ Department of General Medicine, Local Health Unit TO3, Pinerolo \\ (TO); ${ }^{3}$ Department of Microbiology and Virology, University Hospital "Città della Salute e della Scienza di Torino", \\ Turin, Italy
}

\begin{abstract}
Summary
Rhodotorula is emerging as a relevant cause of nosocomial and opportunistic infections. Herein, we present a case of fungaemia due to Rhodotorula mucilaginosa in a cancer patient with lumbosacral stimulator for herniated disc with unfavourable outcome. The patient was hospitalized for twenty days during which he underwent various diagnostic tests before discovering the presence of colon cancer. At day 16 of hospitalization, a bloodstream infection due to $R$. mucilaginosa with an antimycogram profile resistant to fluconazole occurred. It is emphasized the need for the rapid and correct identification of $R$. mucilaginosa in order to set up as fast as possible a pathogen driven therapy, in particular in the immunocompromised subjects.
\end{abstract}

\section{Introduction}

Rhodotorula, Basidiomycota phylum, is a common environmental yeast that is found in air, soil, lakes, ocean water, milk, and

Correspondence: Valentino Granero, S.C. Laboratorio Analisi Unificato Rivoli-Pinerolo ASL TO3, Via B. Cagliari 39, 10064 Pinerolo (TO), Italy. Tel.: +39.0121.233218 - Fax: +39.0121.233034.

E-mail:vgranero@aslto3.piemonte.it

Key words: Rhodotorula mucilaginosa, fungaemia, opportunistic infection.

Contributions: VG, first author, microbiologist during the case report; $\mathrm{EP}$, author, microbiologist during the case report; NM, author, physician during the case report; $\mathrm{NL}$, author, director of medical division during the case report; CC, author, microbiologist consultant/supervisor during the case report; MRC, author, director of laboratory analysis during the case report.

Conflict of interest: the authors declare no potential conflict of interest.

Received for publication: 1 June 2017.

Revision received: 31 August 2017.

Accepted for publication: 3 September 2017.

CC Copyright V. Granero et al., 2017

Licensee PAGEPress, Italy

Microbiologia Medica 2017; 32:6827

doi: $10.4081 / \mathrm{mm} .2017 .6827$

This article is distributed under the terms of the Creative Commons Attribution Noncommercial License (by-nc 4.0) which permits any noncommercial use, distribution, and reproduction in any medium, provided the original author(s) and source are credited. fruit juice. It colonises plants, humans, and other mammals (9).

The genus includes eight species, among which $R$. mucilaginosa (old name $R$. rubra), $R$. glutinis and $R$. minuta are known to cause disease in humans (9). In this regard, in the last two decades these yeasts have emerged as opportunistic pathogens mostly in immunocompromised patients (3).

Recent studies have reported incidence of Rhodotorula fungaemia between $0.5 \%$ and $2.3 \%$ in the USA and Europe (7). Most cases of Rhodotorula fungaemia are associated with the presence of central catheters in patients with haematological malignancies $(2,8)$.

We report a case of $R$. mucilaginosa fungaemia in a cancer patient with lumbosacral stimulator for herniated disc with unfavourable outcome.

\section{Case Report}

The patient was a 69-year old male suffering from herniated disk and unaware of the presence of colon cancer. At admission to the emergency department, the patient presented with fever of $39^{\circ} \mathrm{C}$ lasting three days associated with diarrhoea and difficulty in ambulation. He was admitted to the medicine department for deterioration of general condition with suspected sepsis. The patient presented the followings: heart rate $115 \mathrm{bpm}$, temperature: $37.5^{\circ} \mathrm{C}$, Glasgow Coma Score: 15, rhabdomyolisis, and abnormal haemotochemical analysis (CPK $1877 \mathrm{IU} / \mathrm{L}$, C-reactive protein 34.77 $\mathrm{mg} / \mathrm{dL}, \mathrm{Na}^{+} 132 \mathrm{mEq} / \mathrm{L}$, INR 1.75 ). After the insertion of a urinary catheter, blood (one set) and urine cultures were performed and resulted negative. Subsequently, he was treated with Amoxicillin (e.v. 2.2 g every eight hours in $100 \mathrm{~mL}$ of saline, for three days) and Azithromycin (e.v. $500 \mathrm{mg}$ in $250 \mathrm{~mL}$ of saline, in a single administration), acetaminophen (e.v. 1 vial for three times on day, for three days) and gabapentin (os: $300 \mathrm{mg}$ for two times at day), haloperidol (for agitation), with no effective benefit. Due to the progressive deterioration of motor performance and appearance of cognitive impairment (ideational slowdown, mental confusion, disorientation), at day 10 the patient was transferred to the neurology unit and the following studies were performed: brain computed tomography scan (evidencing soft signs of chronic vascular changes); electroencephalogram (characterized by slow abnormalities on central and front-temporal regions); cerebrospinal fluid examination (clear, 2 leukocytes/ $\mathrm{mm}^{3}$, increase in proteins and albumin, negative culture and neurotropic viruses). On day 12 , given the dubious clinical conditions and after infectious disease consultancy, the therapy was replaced by Meropenem ( $2 \mathrm{~g}$ for 3/die, for three days) and Acyclovir (10 mg pro-kg for 3/die, for three days) with substantial benefit and disappearance of fever.

Two days after discontinuation of antibiotic therapy (day 16 of 
hospitalization), the patient presented again fever $\left(38.8^{\circ} \mathrm{C}\right)$ with tachycardia, dyspnoea and somnolence. The following studies were performed: blood gas analysis, blood tests and culture (two sets of samples spaced 30'); thoracic and abdominal computed tomography, and computed tomography angiography. The presence of solid lesions in the adrenal area, with infiltration of the inferior vena cava and thickening of the rectum was found.

At day 18, colonoscopy confirmed the diagnosis of colon cancer. Meanwhile, preliminary identification of $R$. mucilaginosa from blood cultures performed at day 16 was made. Only both aerobic bottles of blood culture became positive after twenty-nine hours of incubation at $35^{\circ} \mathrm{C}$ (BD BACTECT, Becton Dickinson, Franklin Lakes, NJ, USA); Gram staining evidenced the presence of yeasts. Specimen was seeded on Agar Sabourad (Kima Vacutest, Padua, Italy) and ChromID ${ }^{\circledR}$ Candida (BioMerieux, Mercy L'Etoile, France) after overnight incubation at $37^{\circ} \mathrm{C}$.

The following day, the patient was afebrile in the absence of antifungal therapy. Antimycogram was available in the morning of day 20 (Table 1); in the evening the patient died of neoplastic thrombosis of the inferior vena cava.

\section{Discussion and Conclusions}

The genus Rhodotorula is a pigmented yeast classified under the family Cryptococcaceae and includes 38 species. Rhodotorula species have several morphologic and physiologic similarities with Cryptococcus species, but differ from them by the typical carotenoid pigment (ranging from yellowish to red) (6) and by the inability to assimilate inositol (9).

Members of Rhodotorula species are generally considered to be non-pathogenic and have rarely been a cause of infection in humans. They are commonly recovered from human skin, lungs, conjunctivae, urine and gastrointestinal tract (1). Though it is a

Table 1. Antimycogram susceptibility test.

\begin{tabular}{lcc} 
Drug & MIC $(\mathrm{mg} / \mathrm{mL})$ & Results \\
Itraconazole & 2 & Sensible \\
Voriconazole & 2 & Sensible \\
\hline Fluconazole & $>64$ & Resistant \\
Caspofungin & 2 & Sensible \\
\hline Posaconazole & ND & ND \\
Amphotericin B & ND & ND \\
\hline 5-fluorocytosine & ND & ND \\
\hline
\end{tabular}

ND, Not Determined. Inhibition of disk diffusion test with RPMI agar after incubation at $37^{\circ} \mathrm{C}$ for 24 hours. saprophyte, its isolation from blood cultures and other sterile fluids such as cerebrospinal fluid can be relevant when contamination is ruled out (4). Indeed, Rhodotorula spp. have been implicated as a cause of meningitis, endocarditis, ventriculitis, peritonitis, fungaemia, central venous catheter infection and keratitis (9).

However, bloodstream infection due to Rhodotorula is extremely rare and mostly associated with underlying immunosuppression or cancer $[4,7,8]$.

In this case, there were microbiological evidence of the Rhodotorula presence in blood culture and clinical evidence of neoplasm. Despite the unfavourable outcome, this report highlights the risk of developing invasive fungal infections by Rhodotorula in immunocompromised patients. Antimycogram profile was coherent with those previously reported with the presence of resistance only to fluconazole $[5,7,8]$.

In conclusion, it is emphasized the need for the rapid and correct identification of $R$. mucilaginosa in order to set up as fast as possible a pathogen driven therapy, in particular in the immunocompromised subjects.

\section{References}

1. Duggal S, Hemant J, Amit T, et al. Rhodotorula fungemia: two cases and a brief review. Med Mycol 2011;49:879-82.

2. Garcia-Suarez J, Gomez-Herruz P, Cuadros JA, et al. Epidemiology and outcome of Rhodotorula infection in haematological patients. Mycoses 2011;54:318-24.

3. Miceli MH, Díaz JA, Lee SA. Emerging opportunistic yeast infections. Lancet Infect Dis 2011;11:142-51.

4. Nunes JM, Bizerra FC, Ferreira RC, et al. Molecular identification, antifungal susceptibility profile, and biofilm formation of clinical and environmental Rhodotorula species isolates. Antimicrob Agents Chemother 2013;57:382-9.

5. Pfaller MA, Boyken L, Hollis RJ, et al. Comparison of results of fluconazole and voriconazole disk diffusion testing for Candida spp. with results from a central reference laboratory in the ARTEMIS DISK Global Antifungal Surveillance Program. J Clin Microbiol 2009;47:117-23.

6. Pfaller MA, Dickerna DJ, Merz WG. In: Anaissie EJ, McGinnis MR, Pfaller MA (eds). Infections caused by nonCandida, non-Cryptococcus yeasts. Clinical Mycology. 2nd ed. China: Churchill Livingstone Elsevier, 2009. pp 258-259.

7. Tuon FF, Costa SF. Rhodotorula infection. A systematic review of 128 cases from literature. Rev Iberoam Micol 2008;25:135-40.

8. Tuon FF, Duboc de Almeida GM, Costa SF. Central venous catheter-associated fungemia due to Rhodotorula spp.: a systematic review. Med Mycol 2007;45:441-7.

9. Wirth F, Goldani Z. Epidemiology of Rhodotorula: an emerging pathogen. Interdiscip Perspect Infect Dis 2012;2012:1-7. 